\title{
Multimodel Image Segmentation and Classification by MAP based graph cut and Improved VGG16
}

\author{
Jathin desan
}

\begin{abstract}
Diagnosing brain diseases possess various inbuilt complexities to the nature of the diagnostic process. Brain tumor, Stroke, and Hemorrhage are the commonly prevailing disease and comprise more complexity in diagnosing where there arises the confusion in case of high grade or low-grade tumor and acute or sub-acute stroke. In general most of the prevailing algorithms is suited for the predicted of the image only employing the MRI or CT image. The paper mainly focused on the employment of a suitable proposed algorithm to adopt both the CT and MRI images for precise segmentation and classification. The segmentation algorithm is a map (map a posterior) based graph cut method The segmentation results are compared with the existing methods like (FCM) Fuzzy C Means and KFCM Kernel Fuzzy C Means and it is proved that our proposed system outperformed to the performance metrics. An improved VGG 16architecture is proposed for efficient classification. The overall classification results proved to be more efficient when compared with the existing R-CNN and NS-CNN methods. The paper focused on overcoming the difficulty and make a clear understanding of segmenting and classification irrespective of the nature of the diagnostic process.
\end{abstract}

Keywords: Brain tumor, Hemorrhage, Stroke, VGG 16 architecture, Recurrent Convolutional Neural Network, Graph curt based segmentation, MAP etc.

\section{INTRODUCTION}

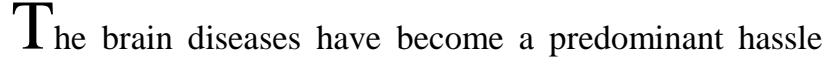
amongst the human beings in which the brain tumor, stroke, and hemorrhage is in most common difficulty [1]. The treatment process greatly depends on the MRI and CT forms of diagnosis for assessing those tumors, stroke and hemorrhage. This diagnostic process restricts manual segmentation in a fully cheap time with accurate quantitative measurements. But growing segmentation methods is a difficult technical process mainly due to the definition of the lesion areas through intensity adjustments. This paper segments and classifies the high grade and low-grade brain tumor image with BRATS 2015 dataset.[2] DWI Diffusion-Weighted Imaging possess a significant role in the brain stroke diagnosis by furnishing elaborative data in case of soft tissue in the brain organ. The paper proposed a segmentation and classification technique for the detection of stoke lesions on the basis of DWI with ISLES dataset by

Revised Manuscript Received on June 05, 2020.

* Correspondence Author

Jathin desan*, 11th grade, in independence school, friscos texas, united states.E-mail: jathindesan95@mail.com

(C) The Authors. Published by Blue Eyes Intelligence Engineering and Sciences Publication (BEIESP). This is an open access article under the CC BY-NC-ND license (http://creativecommons.org/licenses/by-nc-nd/4.0/) using map based Graph cut segmentation technique. [3, 4] BH has been classified as subdural hemorrhage, subarachnoid hemorrhage, intracerebral hemorrhage, and Extradural hemorrhage. In CT imaging, the rotation of the X-ray tube around the patient's head and captures several images. The CT images need an expertized system for precise investigation and analysis [5].The machine learning algorithms which are suitable to train a large number of multi-layer neural networks are engaged for the classification of various automated tasks. The paper focused on overcoming the difficulty and make a clear vicinity for segmenting via map-based graph cut method and classification via improved VGG 16 architecture.

\section{A. Objective}

- To frame an efficient segmentation and classification technique which could apply to both CT and MRI brain images

- To segment, the provided image by map base Graph cut segmentation technique

- To classify the segmented image using improved VGG 16 architecture

\section{B. The organization of the paper}

The continuing subdivision of this paper is prearranged as surveys; Section II clarifies the related works on the segmentation and classification technique. Section III illuminates the implementation of the proposed segmentation and classification. Section IV presents the experimental results of the proposed data collection. Section V shows the performance analysis of the proposed approach. Finally, section VI concluded the proposed segmentation and classifier technique remarks, respectively.

\section{REVIEW OF EXISTING WORKS}

The following represents the survey of the literature regarding the proposed system

[6] implemented the end to end architecture named RescueNet for the segmentation of the brain tumor. The paper overcame the requirement of large scale MRI data (tumor) for deep network training. The study performed discrete training for core, whole and enhance tumor with the three various networks. The system required minimum data for the process of training which could able to provide the best segmentation outcomes on large scale testing data. The scale variant approach for the processing enhances the performance of the network for accurate complete tumor segmentation. The study employed BraTS 2015 and BraTS 2017 databases for brain tumor for assessing the efficiency of the suggested network. DICE coefficient and Sensitivity measure were performed for the comparative analysis.

\section{Published By:}

Blue Eyes Intelligence Engineering

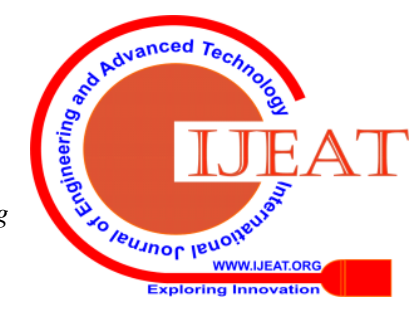


The future perspective of the paper integrates the multitask learning method to improve accuracy. The work also utilized the same framework for other such imaging process and segmentation.[7]employed a technique that fuses the MRI sequence at various levels by utilizing wavelength Transform for obtaining the required data for the detection of the tumor. The images are sent to CNN for the process of instinctive feature extraction and classification. The technique obtained better results on the fused images like BRATS 2012 Image(0.97 ACC), BRATS 2013 Leader board(0.96 ACC), BRATS 2013 Challenge(0.98 ACC), BRATS 2018 Challenge(0.97 ACC), BRATS 2015 Challenge(1.00 ACC) datasets. The task is on the basis of MRI fusion and the future work deals with CT and PET for analyzing the classification results.[8]proposed an assessment method to study the SPES and SISS .The Study includes sixteen groups engaged in the automatic segmentation algorithm. The study made the segmentation feasible for the SPES acute perfusion lesions. But the accuracy is limited when the algorithm was implemented to the sub-acute lesion. The study recommends to optimize the existing algorithm and stated the utilization of isles-challenge as the benchmarking resource.[9]investigated a deep CNN for the segmentation of DWI acute ischemic lesions. The study validated the algorithm on a huge real dataset and proved to be effective when compared with other techniques in terms of average dice coefficient. The combination of MUSCLE Nets + EDD obtained better results with few challenges like semantic segmentation and unavailability of end to end testing and training which reduced the efficiency of the system. The study considered only false positives in the second stage. But few false negatives must also be corrected. The study can generate automatically generate ischemic lesion segmentations. The three dimensional convolutions needs the image patches to be isotropic. But the dataset image slices were simple and thick methods like resampling. And hence the study considered implementing image resolution methods for improving the $3 \mathrm{D}$ images thereby engaging the images on CNN.[10]presented a completely automated segmentation for individual images of the patient without dataset training. The study utilized an EM approach for the estimation of intensity for both pathological and normal issue. The process was performed based on iterative updation with respect to the best voxel intensity. The work resolved the manual levelset initialization by a convex formulation. The efficiency of the technique for the segmentation of ischemic stroke was depicted by the average Dice coefficient of 0.51 and 0.78 for the SISS and SPES 2015 training set.[11]trained three segmentation technique with the use of two dimensional image slices and images achieved in coronal, axial and sagittal aspect respectively and integrate for segmenting the brain tumors with the use of vote based fusion technique. The work segmented the images of brain slice by slice faster than image patches. The assessment was based on the imaging data given by BRATS 2016, BRATS 2013 and BRATS 2015.The results described that our technique performed a segmentation with Flair,T2 and T1C scans and obtained high efficiency.[12] explored a method named EMMA (Ensembles of Multiple Models and Architectures) for high performance by the integration of prediction from various methodologies. The method decreased the impacts of meta parameters and over fitting risk for a particular database. It could be viewed as an unbiased deep learning method for yielding extraordinary performance in BRATS 2017 competition.[13] assessed the suggested technique on BRATS 2013 dataset for obtaining the $1^{\text {st }}$ position on the leaderboard dataset and $2^{\text {nd }}$ position on the challenge dataset. When compared with the state of art methods the suggested technique can obtain high performance with Flair,T2 and T1 C rather than the four imaging modalities Flair,T2, T1c and T1 that decreased the storage and data acquisition cost. The work segmented slice by slice more rapid than by patch by patch. The paper obtained better results with BRATS 2016.[14]presented a deep CNN on the basis of autoencoders with the aim of segmentation of enhanced signal regions. The paper employed BRATS dataset and the performance level estimation algorithm and simultaneous truth was utilized for the provision of ground truth followed by the calculation of Jaccard coefficient, Dice coefficient, false negative fraction and true positive fraction. The suggested technique could be utilized for the production of automatic segmentations with respect to fluid-attenuated inversion recovery regions.[15] investigated the AlexNet, Resnet-18, VGG-16 and ResNet-50 pretrained models for the classification of MR images into cerebrovascular, normal, degenerative, neoplastic and inflammatory divisions. The study predicted that the model ResNet-50 achieved the high accuracy within the five divisions.[16]proposed a cascade model with two CNN for the identification of bleeding and FCN for the detection of five various subtypes of ICH and for the delineation of the lesions. Every CNN-FCN framework were trained individually on the image data that are preprocessed by various settings of window width (default window and stroke window).By their integration, the study achieved efficient results on both segmentation and binary classification of hemorrhagic lesions when compared with all the CNN and FCN model.The study obtained net segmentation efficiency at $80 \%$ accuracy and $82 \%$ recall which is greater than nearly $3.5 \%$ compared with the single FCN model.[17]addressed the issue of $\mathrm{BH}$ identification in the earlier stage of haemorrhage.For solving the problem a CNN approach known as AlexNet integrated with SVM classifier were trained for the brain CT images into the corresponding non-hemorrhage and hemorrhage images. This paper also studied the merits of SVM classifier in the place of three layer neural network. Apart from that the suggested model the AlexNet developed from the scratch outperformed the CNN network.[18]presented a deep learning ICHNet that determines the ICH segmentation. The medical imaging formulated the prediction biased on the basis of the background. The suggested model possessed the capability of training only with the pixels from the brain that enhanced the segmentation performance and optimization time. Further the suggested model decreased the data Skewness by the use of the objective function named dice coefficient. The study relays on the building of 3D ICHNet for various applications with CT and MRI data. 


\section{PROPOSED SYSTEM}

An effective map based graph cut segmentation and classification based on improved VGG 16 architecture system have been briefly explained. The figure 2 represents the overall flow of the proposed system.
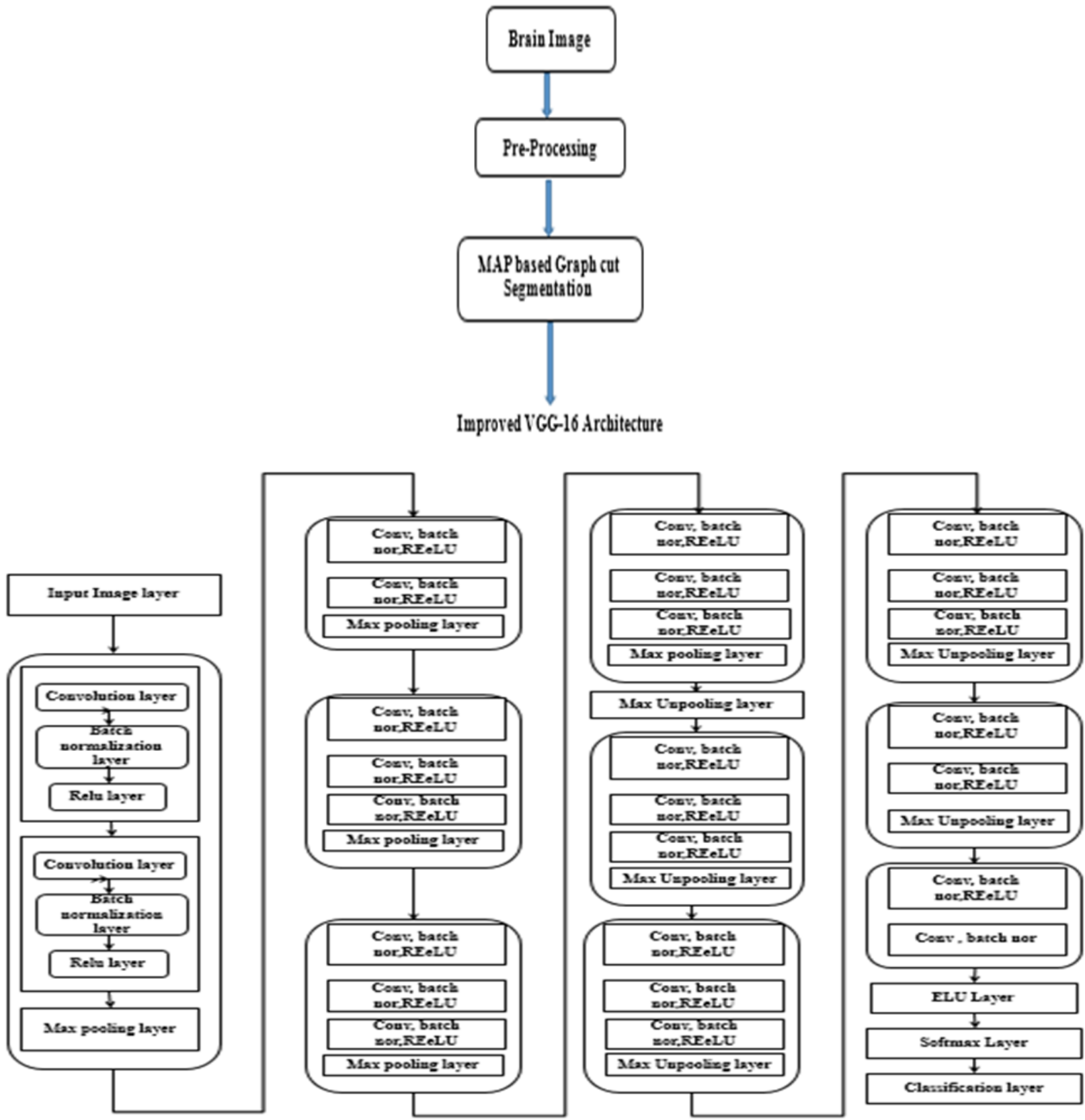

Fig. 1.Overall flow of the proposed system

\section{A. Graph Cut based Segmentation}

Graphical algorithms is now being extensively utilized for resolving the issues related with image segmentation. By considering the super pixel or pixel as a nodal region and allocating an edge to the adjacent pixels or super pixels, graph cut structure could be deployed from the corresponding image. It is followed by the segmentation of the image by the division of the nodal regions into several groups with respect to the some energy minimization criteria. At this stage the segmentation of the image is characteristically framed as a problem of pixel labelling in which various labels represents various group identifiers.

Say $P L_{\text {vee }}$ represent the pixel label vector that is described as

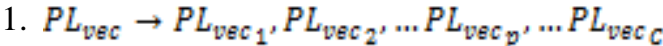

Here $\mathbf{C}$ is the number of image pixels and $\mathrm{PL}_{\text {vec }_{2}}$ represents a pixel label $p$ (that is $\mathrm{PL}_{\text {wec }_{\mathrm{p}}} \neq 0$ for foreground ${ }_{\text {o }} \mathrm{PL}_{\text {vec }_{p}}=0$ for background). The minimized energy function is framed as

Published By: Blue Eyes Intelligence Engineering \& Sciences Publication

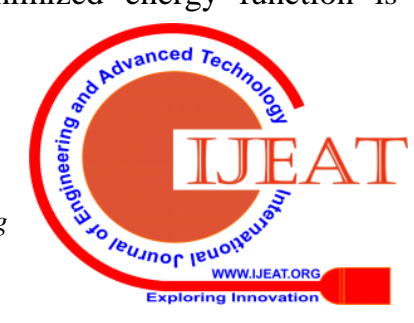


$E\left(P_{\text {vec }}\right)=\tau \sum_{p z P} E_{d t}\left(P_{\text {vec }_{p}}\right)+\sum_{(p, q) \in Y} E_{\text {st }}(p, q) \theta\left(P_{\text {wec }_{p}}, P L_{\text {vec }_{q}}\right)$ in which

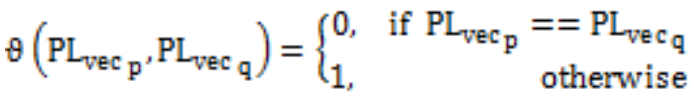

In the second equation, $\gamma$ the neighbourhood system, $P$ is the set of all the pixels, and scalar value $\tau$ denotes the significance of the data term $\mathrm{E}_{\mathrm{dt}}$ with respect to smoothness termE $E_{\text {st }}$. The data term signifies for the label fitness $P L_{\text {vee } p}$ at pixel $p$, which is stated as

$$
\mathrm{E}_{\mathrm{dt}}\left(P L_{\text {wee }} \mathrm{p}\right)=-\ln \mathrm{p}\left(\operatorname{Im}_{\mathrm{p}} \mid P L_{\text {wee }}\right)
$$

Apart from that the colour or intensity at pixel $p_{s} \operatorname{Im}_{p}$ fits into the histogram object or the Gaussian mixture model is calculated as a negative log-likelihood. The smoothness term is utilized to employ the same label for the adjacent pixels with same colours that is stated as

$\mathrm{E}_{\mathrm{st}}(\mathrm{p}, \mathrm{q})=\exp \left(-\frac{\left\|\mathrm{Im}_{\mathrm{p}}-\operatorname{Im}_{\mathrm{q}}\right\|^{2}}{2 \sigma^{2}}\right)$

Here $\sigma$ represents a value of standard deviation

$\left\|\operatorname{Im}_{\mathrm{p}}-\operatorname{Im}_{\mathrm{q}}\right\|$ is the Euclidean distance found in-between the two colour vectors.

This algorithm was extensively utilized with interactive segmentation in which the used given seeds are utilized in describing the data term. The colour image segmentation could be achieved by consolidating the equation. The depth and colour of the kinetics were observed for obtaining the image pairs. The proposed system finds the value of the standard deviation by a novel technique called map which is known as map a posterior. The obtained $\sigma$ value should be very small for high efficiency and it denotes the shape prior.

\section{B. Proposed}

Let $B_{\text {map }}$ represent a binary map with the same color image size whose value is 1 at pixel $p$ if $p$ is on one of the lines joining the joints of the $n t h$ object. The Bayes rule stated that

$p\left(P L_{\text {vec }}=\mathrm{n} \| \operatorname{Im}_{\mathrm{x}}\right) \propto p\left(\operatorname{Im}_{\mathrm{x}} \mid P L_{\text {vec }}=\mathrm{n}\right) p\left(P L_{\text {vec } p}=\mathrm{n}\right.$ . Here $p\left(P L_{\text {vee } p}=\mathrm{n}\right)$ is a corresponding probability of $p$ being labelled asn, which is denoted as

$$
p\left(P L_{\text {vec } p}=\mathrm{n}\right)=\exp \left(-\frac{D_{\text {dist }}^{2}\left(p_{i} B_{\text {map }} n\right.}{2 \sigma_{J}^{2}}\right)
$$

In which $\sigma J$ is the standard deviation and $D_{\text {dist }}^{2}\left(p_{v} B_{\text {map }_{n}}\right)$ is the distance found between $p$ and $B_{\text {map }}$ $P L_{\text {vecp }}$ is the distance found between the vector $\mathrm{p}$ and the nearest pixel $B_{\text {map }}$

$$
p\left(P L_{\text {vec } p}=0\right)=1-\underset{n \in\{1,2, \ldots M\}}{\max } p\left(P L_{\text {wec } p}=\mathrm{n}\right)
$$$$
\mathrm{E}_{\mathrm{dt}}\left(P L_{\text {Vee }} \mathrm{p}\right)=-\ln \mathrm{p}\left(\operatorname{Im}_{\mathrm{p}} \mid P L_{\text {Vec }}\right)-\ln \mathrm{p}\left(P L_{\text {Wee }}\right)
$$

In spite of video segmentation with three dimensional graph structure, the method is computationally more expensive and memory consuming. The study observed that the frame by frame segmentation is sufficiently enough for brain segmentation with respect to the shape prior which leads to shape prior given by the depth image.

\section{Improved VGG -16 architecture}

VGG 16 is a CNN which had been trained on million number of images from the corresponding image Net databases. It is a sixteen layer deep network that classified the images into thousand object categories. And hence the network possess an input image size of 224 by 224 . The architecture comprised of 41 layers that includes 16 layers (learnable weights), 3 fully connected layers and 13 convolution layers.

Our study changed the 37th ReLU layer into leaky ReLU layer in which the alpha value was found by Chicken Swarm Optimization algorithm. The leaky ReLU layer was created under the name 'leaky 1' followed by a scalar multiplier for negative input which is equal to 0.1.It included the layer in 11 layer arrays.

The flow depicted is being explained below. The brain image to be examined is subjected to preprocessing followed by MAP based Graph cut segmentation method. After the process of segmentation the study performed improved VGG 16 architecture which is described below in detail.

\section{PERFORMANCE ANALYSIS}

The performance analysis have been depicted below

\section{A. Tumour}

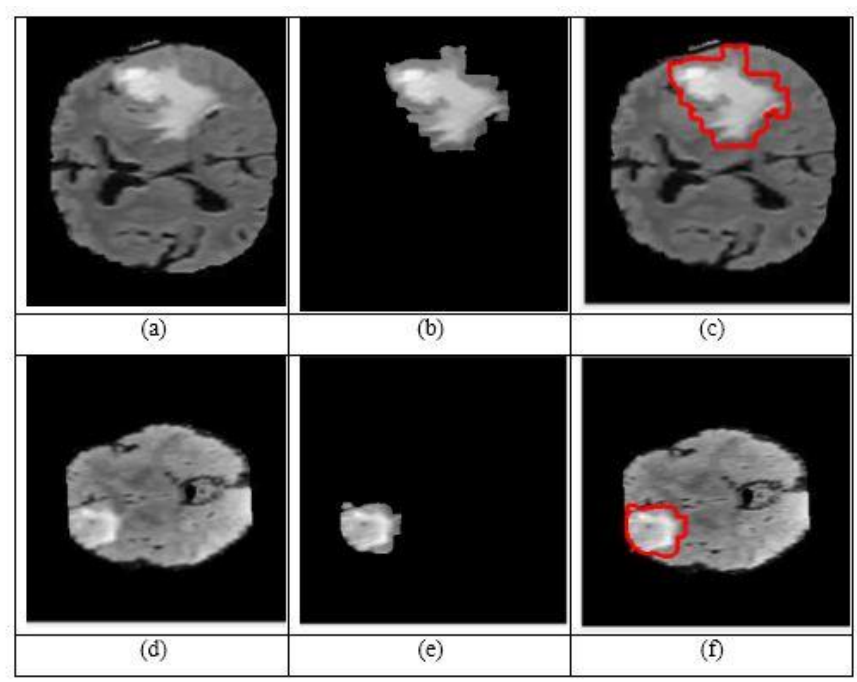

Figure 3 Labelled tumor image

\section{B. Stroke}

Published By: Blue Eyes Intelligence Engineering $\&$ Sciences Publication

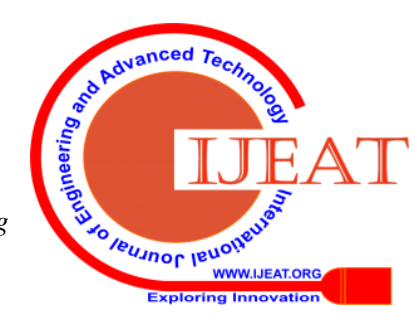



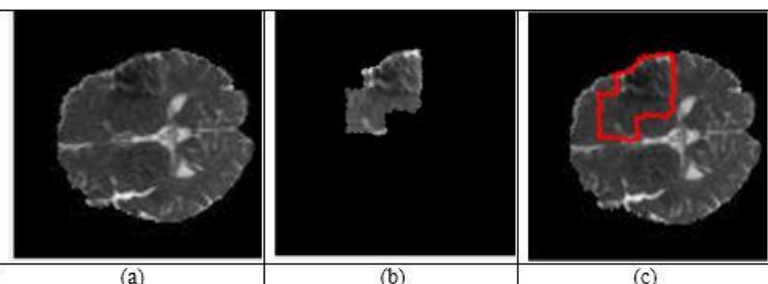

(b)
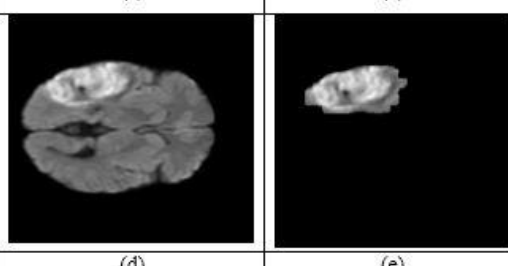

(c)

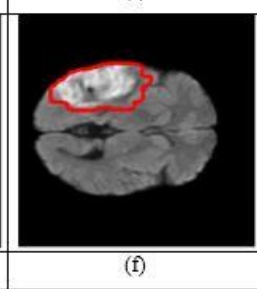

Figure 4 Labelled stroke image

The figure 4 represents the labeled image with the help of masking operation for performing the stroke segmentation operation on the brain DWI MRI and CT. The scans with the masked ground truth are arranged to obtain the non-paired training for complete stroke segmentation. To make our study more precise towards segmentation, the segmented region with the original pixels were created. Finally the segmented region is enhanced with the highlighted original pixel region to depict a more clear result. In the figure 3, (a) represented the input acute tumor image (b) represented the segmented region with original pixel, the figure (c) is the enhanced segmented image, (d) represented the input sub-acute image (e) represented the segmented region with original pixel and the figure (f) is the enhanced segmented image.

\section{HEMORRHAGE}
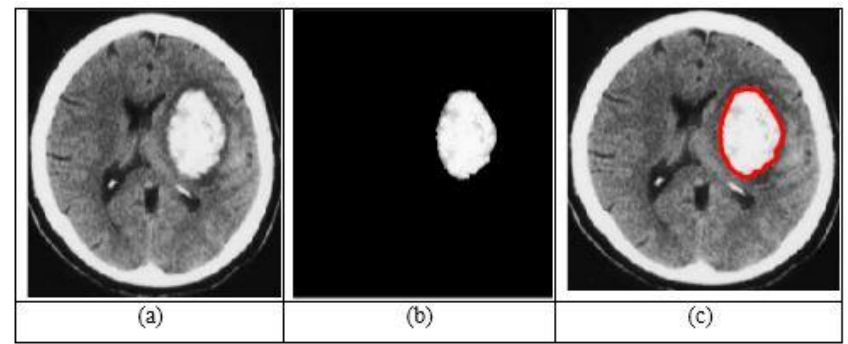

Figure 3 Labelled Hemorrhage image

The figure 5 represents the hemorrhage image (a) represented the input image (e) represented the segmented region with original pixel of the hemmorrhage and the figure (c) is the enhanced segmented hemmorrhage image.

The following demonstrates the segmentation and classification results with respect to the performance metrics such as Sensitivity, Specificity, recall, Jaccard_coefficient, Dice_coefficient, kappa_coefficient, Accuracy, RI, GCE, VOIb for tumor,stroke and hemorrhage.

\section{Segmentation results}

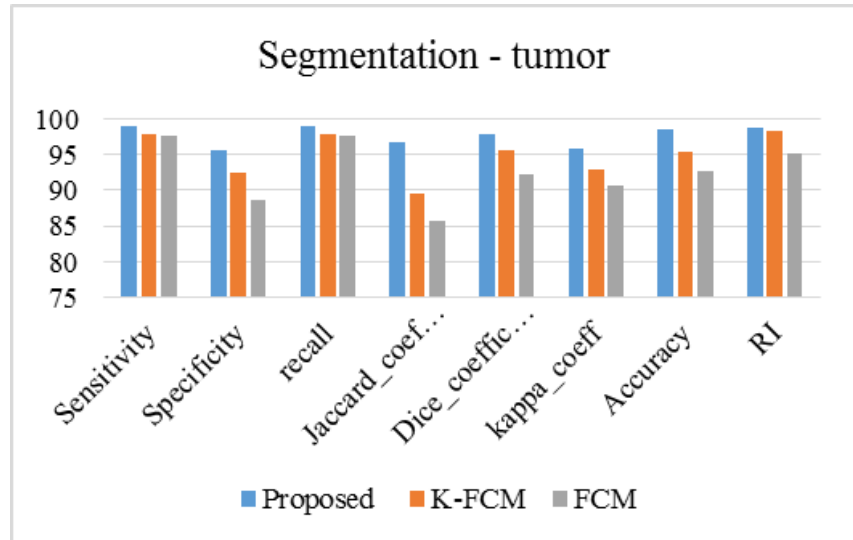

Figure 6: Performance analysis of proposed segmentation results for tumor

Figure 6 depicts the various performance analysis of proposed method segmentation results for tumor. The performance analysis are sensitivity, specificity, recall, Jaccard co-efficient, kappa-coefficient, accuracy and rand index. The proposed method provides the accuracy of $98.57 \%$ and specificity of $95.69 \%$, sensitivity of $98.89 \%$ and recall contains $98.89 \%$. These proposed method results are compared with existing K-FCM and FCM methods. This analysis proved that the proposed method had highest accuracy compared than existing methods.

Table I: Segmentation results of proposed method for tumor

\begin{tabular}{|c|c|c|c|}
\hline $\begin{array}{l}\text { Performance } \\
\text { metrics }\end{array}$ & $\begin{array}{l}\text { Propose } \\
\text { d }\end{array}$ & K-FCM & FCM \\
\hline VOI & 0.325 & 0.895 & 1.89 \\
\hline GCE & 2.89 & 3.89 & 14.27 \\
\hline
\end{tabular}

Table 1describes the segmentation analysis of tumor for proposed method and existing methods K-FCM and FCM with the performance measures of Variation of Information and Global Consistency Error (GCE). The proposed method had $0.325 \%$ of VOI and $2.89 \%$ of GCE. This anlaysis proved that the proposed method contains minimum rate of error compared than existing methods.

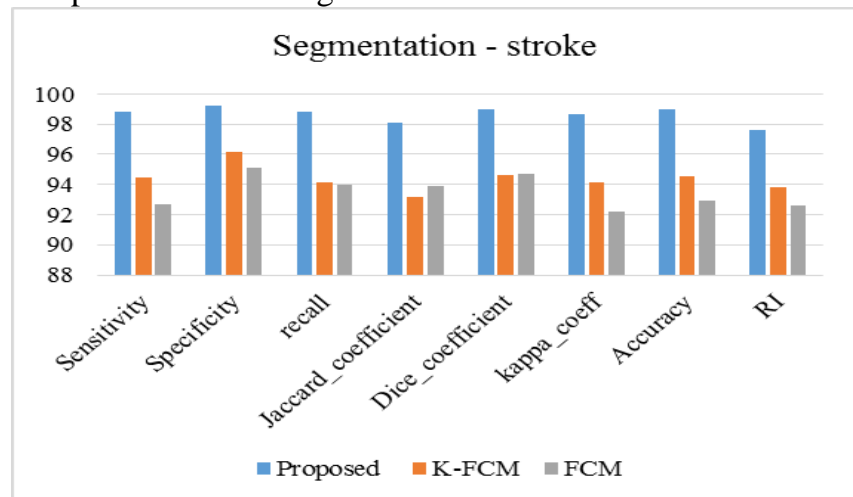

Figure 7: Performance analysis of proposed segmentation results for stroke

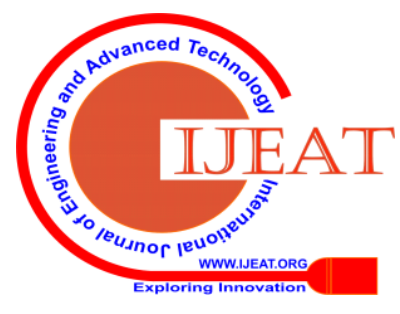


Figure 7 depicts the various performance analysis of proposed method segmentation results for stroke. The performance analysis are sensitivity, specificity, recall, Jaccard co-efficient, kappa-coefficient, accuracy and rand index. The proposed method provides the accuracy of $99.02 \%$ and specificity of $99.23 \%$, sensitivity of $98.82 \%$ and recall contains 98.82\%. These proposed method results are compared with existing K-FCM and FCM methods. This analysis proved that the proposed method had highest accuracy compared than existing methods.

Table 2: Segmentation results of proposed method for stroke

\begin{tabular}{|l|r|r|r|}
\hline $\begin{array}{l}\text { Performance } \\
\text { metrics }\end{array}$ & $\begin{array}{l}\text { Propose } \\
\mathrm{d}\end{array}$ & K-FCM & \multicolumn{1}{c|}{ FCM } \\
\hline GCE & 2.33 & 3.98 & 15.5206 \\
\hline VOI & 0.423 & 1.58 & 4.3589 \\
\hline
\end{tabular}

Table 2 defines the segmentation analysis of Stroke for proposed method and existing methods K-FCM and FCM with the performance measures of Variation of Information and Global Consistency Error (GCE). The proposed method had $0.423 \%$ of VOI and $2.33 \%$ of GCE. This analysis proved that the proposed method contains minimum rate of error compared than existing methods.

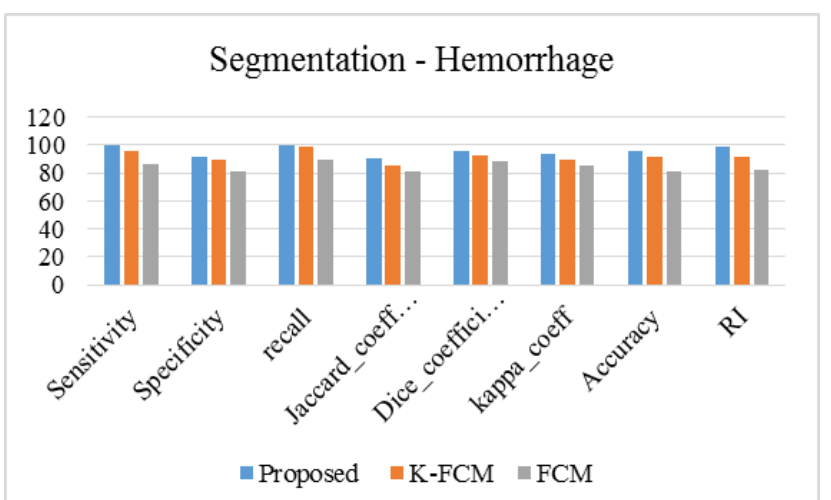

Figure 8: Performance analysis of proposed segmentation results for Hemorrhage

Figure 8 depicts the various performance analysis of proposed method classification results for Hemorrhage. The performance analysis are sensitivity, specificity, recall, Jaccard co-efficient, kappa-coefficient, accuracy and rand index. The proposed method provides the accuracy of 95.03\% and specificity of $91.42 \%$, sensitivity of $99.98 \%$ and recall contains 99.98\%. These proposed method results are compared with existing K-FCM and FCM methods. This analysis proved that the proposed method had highest accuracy compared than existing methods.

Table 3: Segmentation results of proposed method for Hemorrhage

\begin{tabular}{|c|c|c|c|}
\hline $\begin{array}{l}\text { Performance } \\
\text { metrics }\end{array}$ & $\begin{array}{l}\text { Propose } \\
\text { d }\end{array}$ & K-FCM & FCM \\
\hline VOI & 0.458 & 1.235 & 3.58 \\
\hline GCE & 1.01 & 5.89 & 12.05 \\
\hline
\end{tabular}

Table 3 represents the segmentation analysis of Hemorrhage for proposed method and existing methods K-FCM and FCM with the performance measures of Variation of Information and Global Consistency Error (GCE). The proposed method had $0.458 \%$ of VOI and $1.01 \%$ of GCE. This analysis proved that the proposed method contains minimum rate of error compared than existing methods.

\section{E. Classification results}

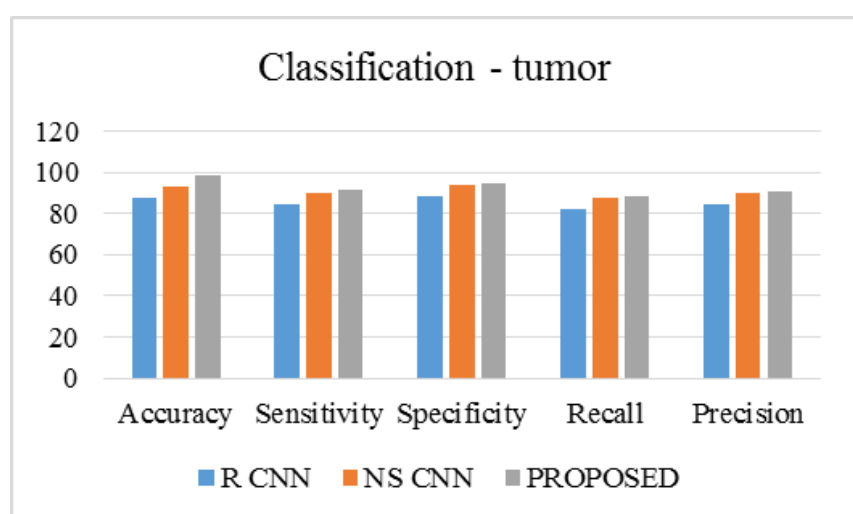

Figure 9: Performance analysis of proposed classification results for tumor

Figure 9 depicts the various performance analysis of proposed method classification results for tumor. The performance analysis are accuracy, sensitivity, specificity, recall, and precision. The proposed method provides the accuracy of $98.26 \%$ and specificity of $94.41 \%$, sensitivity of $91.03 \%$, recall contains $88.19 \%$ and precision value contains $90.63 \%$. These proposed method results are compared with existing R-CNN, and NS-CNN methods. This analysis proved that the proposed method had highest accuracy compared than existing methods.

Table 4: Classification results of proposed method for tumor

\begin{tabular}{|l|c|c|r|}
\hline Performance metrics & R CNN & \multicolumn{1}{|l|}{ CNN } & PROPOSED \\
\hline Jaccard Coefficient & 0.7811 & 0.8371 & 0.9435 \\
\hline F-score & 0.8311 & 0.8871 & 0.9591 \\
\hline Missed Classification & 0.1165 & 0.0478 & 0.0256 \\
\hline
\end{tabular}

Table 4 represents the classification analysis of tumor for proposed method and existing methods R-CNN, and NS-CNN with the performance measures of Jaccard co-efficient, f-score, and missed classification. The proposed method had $0.7811 \%$ of Jaccard co-efficient, $0.8311 \%$ of F-score and $0.1165 \%$ of missed classification. This analysis proved that the proposed method contains minimum rate of error compared than existing methods.

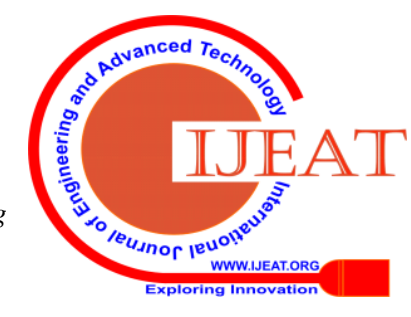




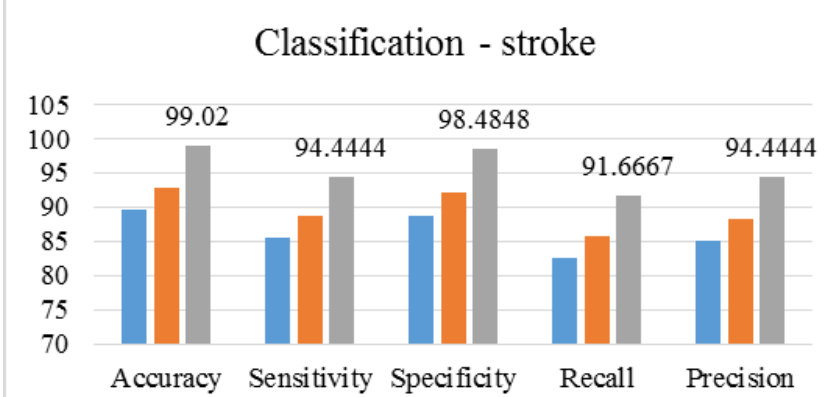

$\mathrm{RCNN}=\mathrm{NSCNN} \square \mathrm{PROPOSED}$

Figure 10: Performance analysis of proposed segmentation results for stroke

Figure 10 depicts the various performance analysis of proposed method classification results for stroke. The performance analysis are accuracy, sensitivity, specificity, recall, and precision. The proposed method provides the accuracy of $99.02 \%$ and specificity of $94.44 \%$, sensitivity of $98.48 \%$, recall contains $91.66 \%$ and precision value contains 94.44\%. These proposed method results are compared with existing R-CNN, and NS-CNN methods. This analysis proved that the proposed method had highest accuracy compared than existing methods.

Table 5: Classification results of proposed method for stroke

\begin{tabular}{|l|r|r|r|}
\hline $\begin{array}{l}\text { Performance } \\
\text { metrics }\end{array}$ & R CNN & NS CNN & PROPOSED \\
\hline $\begin{array}{l}\text { Jaccard } \\
\text { Coefficient }\end{array}$ & 0.8875 & 0.9191 & 0.8611 \\
\hline F-score & 0.9031 & 0.9347 & 0.9111 \\
\hline $\begin{array}{l}\text { Missed } \\
\text { Classification }\end{array}$ & 0.0943 & 0.0526 & 0.0278 \\
\hline
\end{tabular}

Table 5 represents the classification analysis of tumor for proposed method and existing methods R-CNN, and NS-CNN with the performance measures of Jaccard co-efficient, f-score, and missed classification. The proposed method had $0.8875 \%$ of Jaccard co-efficient, $0.9031 \%$ of F-score and $0.0943 \%$ of missed classification. This analysis proved that the proposed method contains minimum rate of error compared than existing methods.

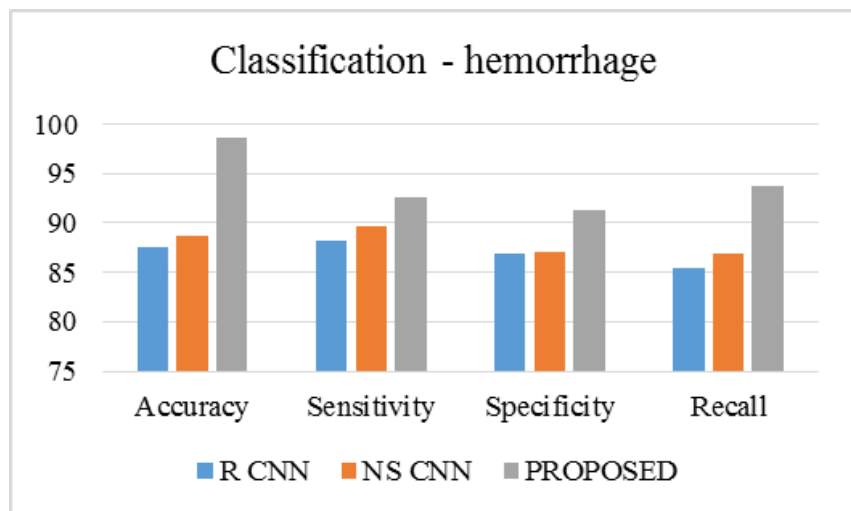

Figure 11: Performance analysis of proposed segmentation results for hemorrhage

Figure 11 depicts the various performance analysis of proposed method classification results for hemorrhage. The performance analysis are accuracy, sensitivity, specificity, recall, and precision. The proposed method provides the accuracy of $98.57 \%$ and specificity of $92.56 \%$, sensitivity of $91.23 \%$, and recall contains $93.78 \%$. These proposed method results are compared with existing R-CNN, and NS-CNN methods. This analysis proved that the proposed method had highest accuracy compared than existing methods.

Table 6: Classification results of proposed method for Hemorrhage

\begin{tabular}{|l|r|r|r|}
\hline Performance metrics & \multicolumn{1}{|c|}{ R CNN } & \multicolumn{1}{l|}{ CNN } & PROPOSED \\
\hline Jaccard Coefficient & 0.7635 & 0.7856 & 0.81 \\
\hline F-score & 0.8123 & 0.8256 & 0.9456 \\
\hline Missed Classification & 0.215 & 0.125 & 0.014 \\
\hline
\end{tabular}

Table 6 represents the classification analysis of tumor for proposed method and existing methods R-CNN, and NS-CNN with the performance measures of Jaccard co-efficient, f-score, and missed classification. The proposed method had $0.7635 \%$ of Jaccard co-efficient, $0.8123 \%$ of F-score and $0.215 \%$ of missed classification. This analysis proved that the proposed method contains minimum rate of error compared than existing methods.

\section{CONCLUSION}

The paper mainly focused on the employment of a suitable proposed algorithm to adopt both the CT and MRI images for precise segmentation and classification. The segmentation algorithm is a map (map a posterior) based graph cut method The segmentation results are compared with the existing methods like (FCM) Fuzzy C Means and KFCM Kernel Fuzzy C Means and it is proved that our proposed system outperformed with respect to the performance metrics. An improved VGG 16 architecture is proposed for efficient classification. The overall classification results proved to be more efficient when compared with the existing R-CNN and NS-CNN methods. The paper focused on overcoming the difficulty and make a clear understanding in segmenting and classification irrespective of the nature of the diagnostic process.

\section{REFERENCES}

1. V. Babu, "Brian tumor segmentation and classification using neural networks in MRI images," 2019.

2. N. Noor, N. Saad, A. Abdullah, and N. Ali, "Automated segmentation and classification technique for brain stroke," International Journal of Electrical and Computer Engineering, vol. 9, p. 1832, 2019.

3. R. M. Sarmento, F. F. Vasconcelos, P. P. Rebouças Filho, W. Wu, and V. H. C. De Albuquerque, "Automatic Neuroimage Processing and Analysis in Stroke-A Systematic Review," IEEE reviews in biomedical engineering, 2019.

4. M. D. Hssayeni, M. S. Croock, A. Al-Ani, H. F. Al-khafaji, Z. A Yahya, and B. Ghoraani, "Intracranial Hemorrhage Segmentation Using Deep Convolutional Model," arXiv preprint arXiv:1910.08643, 2019.

5. M. R. Arbabshirani, B. K. Fornwalt, G. J. Mongelluzzo, J. D. Suever, B. D. Geise, A. A. Patel, et al., "Advanced machine learning in action: identification of intracranial hemorrhage on computed tomography scans of the head with clinical workflow integration," npj Digital Medicine, vol. 1, p. 9, 2018/04/04 2018.

6. S. Nema, A. Dudhane, S. Murala, and S. Naidu, "RescueNet: An unpaired GAN for brain tumor segmentation," Biomedical Signal 
Processing and Control, vol. 55, p. 101641, 2020.

7. J. Amin, M. Sharif, N. Gul, M. Yasmin, and S. A. Shad, "Brain tumor classification based on DWT fusion of MRI sequences using convolutional neural network," Pattern Recognition Letters, vol. 129, pp. 115-122, 2020.

8. O. Maier, B. H. Menze, J. von der Gablentz, L. Häni, M. P. Heinrich, M. Liebrand, et al., "ISLES 2015-A public evaluation benchmark for ischemic stroke lesion segmentation from multispectral MRI," Medical image analysis, vol. 35, pp. 250-269, 2017.

9. L. Chen, P. Bentley, and D. Rueckert, "Fully automatic acute ischemic lesion segmentation in DWI using convolutional neural networks," NeuroImage: Clinical, vol. 15, pp. 633-643, 2017.

10. T. Haeck, F. Maes, and P. Suetens, "ISLES challenge 2015: Automated model-based segmentation of ischemic stroke in MR images," in BrainLes 2015, 2015, pp. 246-253.

11. X. Zhao, Y. Wu, G. Song, Z. Li, Y. Zhang, and Y. Fan, "A deep learning model integrating FCNNs and CRFs for brain tumor segmentation," Medical image analysis, vol. 43, pp. 98-111, 2018.

12. K. Kamnitsas, W. Bai, E. Ferrante, S. McDonagh, M. Sinclair, N. Pawlowski, et al., "Ensembles of multiple models and architectures for robust brain tumour segmentation," in International MICCAI Brainlesion Workshop, 2017, pp. 450-462.

13. X. Zhao, Y. Wu, G. Song, Z. Li, Y. Fan, and Y. Zhang, "Brain tumor segmentation using a fully convolutional neural network with conditional random fields," in International Workshop on Brainlesion: Glioma, Multiple Sclerosis, Stroke and Traumatic Brain Injuries, 2016, pp. 75-87.

14. P. Korfiatis, T. L. Kline, and B. J. Erickson, "Automated segmentation of hyperintense regions in FLAIR MRI using deep learning," Tomography, vol. 2, p. 334, 2016.

15. M. Talo, O. Yildirim, U. B. Baloglu, G. Aydin, and U. R. Acharya, "Convolutional neural networks for multi-class brain disease detection using MRI images," Computerized Medical Imaging and Graphics, vol. 78, p. 101673, 2019.

16. J. Cho, K.-S. Park, M. Karki, E. Lee, S. Ko, J. K. Kim, et al., "Improving Sensitivity on Identification and Delineation of Intracranial Hemorrhage Lesion Using Cascaded Deep Learning Models," Journal of digital imaging, vol. 32, pp. 450-461, 2019.

17. A. M. Dawud, K. Yurtkan, and H. Oztoprak, "Application of Deep Learning in Neuroradiology: Brain Haemorrhage Classification Using Transfer Learning," Computational Intelligence and Neuroscience, vol. 2019, 2019.

18. M. Islam, P. Sanghani, A. A. Q. See, M. L. James, N. K. K. King, and H. Ren, "ICHNet: Intracerebral Hemorrhage (ICH) Segmentation Using Deep Learning," in International MICCAI Brainlesion Workshop, 2018, pp. 456-463.

\section{AUTHORS PROFILE}

Jathin desan 11th grade in independence school, Frisco's Texas, united states.E-mail: jathindesan95@mail.com 